\title{
A COMPARISON BETWEEN MD- AND EXAFS-EXTRACTED STRUCTURAL DATA FOR TERNARY $\operatorname{RbBr}_{1-\mathrm{x}} \mathbf{I}_{\mathrm{x}}$
}

\author{
JAROS $Ł A W$ BOSKO ${ }^{1}$, JAROS $Ł A W ~ R Y B I C K I^{1,2}$, AGNIESZKA WITKOWSKA ${ }^{1,2}$, EMILIANO PRINCIPI ${ }^{3}$ \\ ${ }^{1}$ Faculty of Applied Physics and Mathematics, Gdanisk University of Technology, \\ Narutowicza 11/12, 80-952 Gdańsk, Poland \\ ${ }^{2}$ TASK Computer Centre, Gdańsk University of Technology, \\ Narutowicza 11/12, 80-952 Gdańsk, Poland \\ ${ }^{3}$ INFM UdR Camerino, Istituto di Fisica, Universita di Camerino, \\ via Madonna delle Careen, Camerino (MC), Italia
}

\begin{abstract}
The structure of ternary salts of the composition $\operatorname{RbBr}_{1-x} \mathrm{I}_{x}, x=0.0,0.1,0.2,0.4,0.6,0.8,0.9$ and 1.0 is discussed. In particular, a comparison is made of the structural results obtained from an X-ray absorption spectroscopy (XAS) experiment and the corresponding data obtained in classical molecular dynamics (MD) simulations. In MD simulations, realized in the isobaric-isothermal (NpT) ensemble, the Dixon and Sangster's potential parameterisation (M. Dixon and M. J. L. Sangster, J. Phys. C: Solid State Phys., 9, 3381 (1976)) has been applied. The ability of the theoretical model of interatomic interaction to reproduce the structural properties observed in the EXAFS experiment is critically discussed.
\end{abstract}

\section{INTRODUCTION}

$\mathrm{RbBr}$ and $\mathrm{RbI}$ are examples of typical ionic crystals. It is a marked characteristic of these compounds that they are completely miscible in both their liquid and solid phases. Due to the same crystal structure (that of rock-salt), similar ionic sizes and ionicity, while mixed, they form a crystalline solid solution of the composition $\operatorname{RbBr}_{1-x} \mathrm{I}_{x}$ for the entire composition range. This has been confirmed by X-ray diffraction experiments [1] which indicate that the lattice constant of the ternary compound varies linearly as a function of the composition $x$ (satisfying Vegard's law).

Both extended X-ray absorption fine structure spectroscopy experiments (EXAFS) and classical molecular dynamics simulations (MD) allow one to obtain more profound insight into the local structure of the studied material. Detailed analysis of the first peak of the partial distribution functions, obtained through the EXAFS experiment or MD simulation, permits to observe structural disorder and deviations from harmonic behaviour. Of special interest are average bond lengths $R$, width of the first peak $\sigma$, its asymmetry $\beta$, and the way they depend on the compound stoichiometry.

The opportunity to analyze separately the local structure of ions of different kinds allows one to observe the variation of the average structural properties of the $\mathrm{Rb}-\mathrm{I}$ and $\mathrm{Rb}$ - $\mathrm{Br}$ bonds as a function of the compound composition. Predicted by analytical theories [2-4], the existence of two distinct bond lengths for the pairs has been confirmed experimentally by Mikkelsen and 
Boyce [5, 6] using the EXAFS technique. Advanced analysis of EXAFS signals with the aid of the GNXAS package [7-9] has also made it possible to study with high accuracy bond length variance $\sigma^{2}$ and the asymmetry parameter $\beta$ which give insight into the thermal- and composition-dependent structural disorder.

\section{EXPERIMENTAL METHOD}

An EXAFS experiment on $\operatorname{RbBr}_{1-x} \mathrm{I}_{x}$ has been performed at the BM29 beamline of the European Synchrotron Radiation Facility (ESRF). Low-noise XAS data have been collected for the solid phase at $\mathrm{Rb}, \mathrm{Br}$ and I K-edges at room temperature $(T-300 \mathrm{~K})$. An advanced experimental setup and multiple-edge data analysis techniques have been applied to collect and analyze the spectra. These made possible a high resolution reconstruction of the $\mathrm{Rb}-\mathrm{Br}$ and $\mathrm{Rb}-\mathrm{I}$ nearest neighbour bond length distributions as a function of composition $x$. The EXAFS data have been analysed using advanced ab-initio methods and simultaneous multiple-edge structural refinement implemented in the GNXAS data-analyzing package [7-9]. A detailed description of the experimental technique and the obtained results are published elsewhere [1]. In this contribution, the stoichiometry-dependent values of some parameters describing the shape of the first peak of the ionic partial radial distribution functions are cited in order to be compared to those derived from the results of the MD simulations of the considered compounds.

\section{SIMULATION TECHNIQUE}

Classical MD simulations have been applied to study the structures of the relevant compounds. Both $\mathrm{RbBr}$ and $\mathrm{Rbl}$ compounds in the solid phase, at the room temperature and under atmospheric pressure exist in the rock salt structure, hence solid solutions of the two also appear in the $\mathrm{NaCl}$ structure. Modeled samples were composed of cubic elementary cells repeated periodically 8 times in each direction. In this way a simulation box of the approximate size of $60 \AA$ was build, consisting of 4096 ions. In order to avoid effects associated with the surfaces and small size of the sample, periodic boundary conditions have been applied. In the case of ternary compounds $\mathrm{Br}$ and I ions were homogeneously distributed over the available anion sites.

The ions were represented using the rigid-ion model. To describe the effective inter-ionic interactions, the pairwise Born-Mayer-Huggins type potential has been applied:

$$
\varphi_{i j}\left(r_{i j}\right)=\frac{Z_{i} Z_{j} e^{2}}{4 \pi \varepsilon_{0} r_{i j}}+A_{i j} \exp \left[B_{i j}\left(\sigma_{i}+\sigma_{j}-r_{i j}\right)\right]-\frac{C_{i j}}{r_{i j}^{6}}-\frac{D_{i j}}{r_{i j}^{8}}
$$

where $Z_{i}, Z_{j}$ - the effective ionic charges; $A_{i j}, B_{i j}$ - the Pauling factors; $\sigma_{i}, \sigma_{j}$ - ionic radii; $C_{i j}$, $D_{i j}$ - the van der Waals coefficients. The applied numerical values of the parameters for binary salts were those derived by Dixon and Sangster [10], The Lorentz-Berthelot mixing rule has been applied to achieve parameterisation of ternary compounds. 
The MD simulations were performed in an isobaric-isothermal ensemble (Nosé-Hoover ensemble). The systems were assumed to remain at the constant temperature $T=300 \mathrm{~K}$, under pressure $p=0 \mathrm{~Pa}$. The equations of motion were integrated with the discrete timestep $\Delta t=1 \mathrm{fs}$. The integration Verlet leapfrog scheme was applied. During the first 5000 timesteps the systems were equilibrated, and over further 20000 timesteps the particles' trajectories were monitored. The calculations were performed using the parallel version of the DL_POLY program [11],

To analyze the structure of the studied systems, partial radial distribution functions (RDF) have been extracted:

$$
g(r)=\frac{1}{\rho^{2}}\left\langle\sum_{i=1}^{N} \sum_{j \neq i}^{N} \delta\left(r-r_{i j}\right)\right\rangle,
$$

where $N$ is the number of ions, $\rho$ - partial concentration, and $<\ldots>$ indicates averagaging over time. To describe quantitatively the obtained correlation functions, $\Gamma$-like profiles have been used:

$$
f(r)=\frac{2 N_{c}}{\sigma|\beta| \Gamma\left(\frac{4}{\beta^{2}}\right)}\left[\frac{4}{\beta^{2}}+\frac{2(r-R)}{\beta \sigma}\right]\left(\frac{4}{\beta^{2}}-1\right) \exp \left[-\left(\frac{4}{\beta^{2}}+\frac{2(r-R)}{\beta \sigma}\right)\right],
$$

where $N_{\mathrm{c}}$ - co-ordination number, $R$ - average distance, $\sigma$ - standard deviation, $\beta$ - skewness parameter.

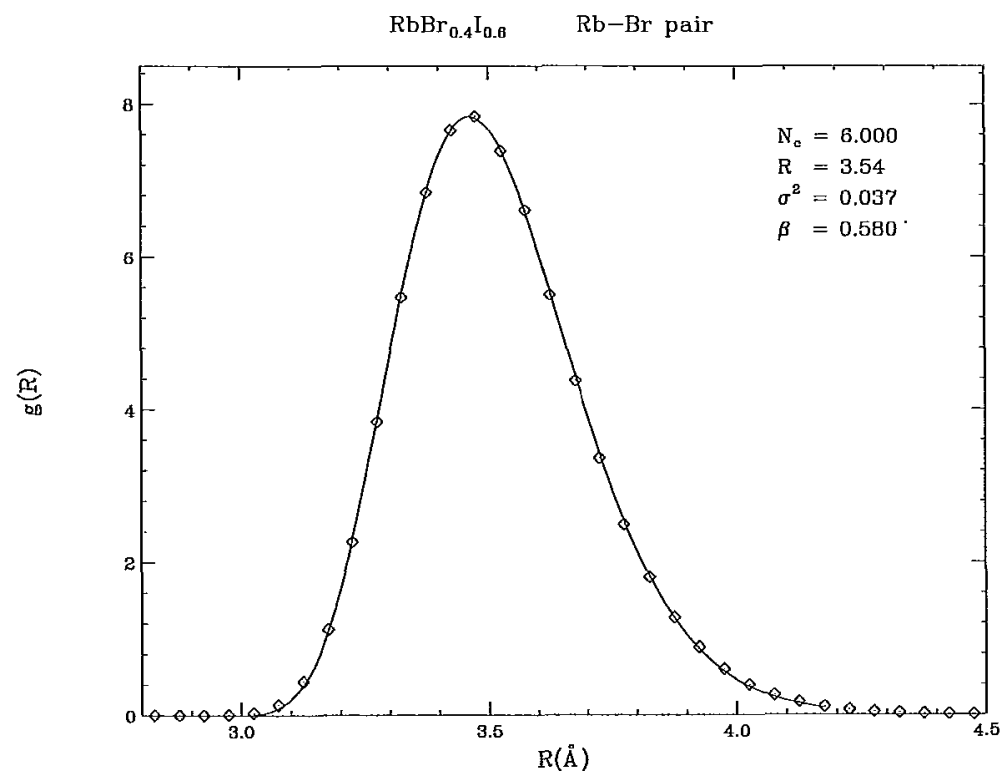

Fig. 1. Example of $\Gamma$-like profile fitting to a $g(r)$ obtained in the MD simulation. The Figure refers to the $\mathrm{Rb}-\mathrm{Br}$ pair correlation in $\mathrm{RbBr}_{04} \mathrm{I}_{06}$ salt. Diamonds - MD simulation, solid line - the best fit $\Gamma$-like function

An example of the $\Gamma$-like representation of the first RDF peak is shown in Fig. 1. A comparison of the obtained parameter values with the experimental data is presented in the subsequent section. 


\section{RESULTS AND DISCUSSION}

Cation-anion RDFs referring to the studied systems have been analyzed. By fitting a $\Gamma$-like profile, the values of the characteristic parameters have been derived. As the structure of all the alloys is the same (the rock-salt structure), in each case the anions are six-fold co-ordinated with $\mathrm{Rb}$, whereas the average co-ordination of $\mathrm{Rb}$ ions is $6 x$ in the case of $\mathrm{Br}$ ions and $6(1-x)$ in the case of I ions, respectively.

The dependence of the average distance between cation-anion pairs $R$, the width of the peak (standard deviation $\sigma$ ) and its asymmetry $\beta$ on the system stoichiometry is presented in Fig. 2. In each case, molecular dynamics data are compared to EXAFS-extracted experimental ones.

EXAFS

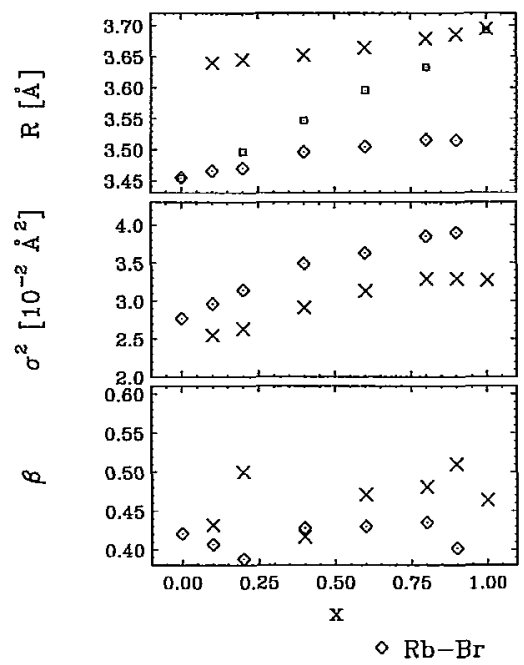

MD

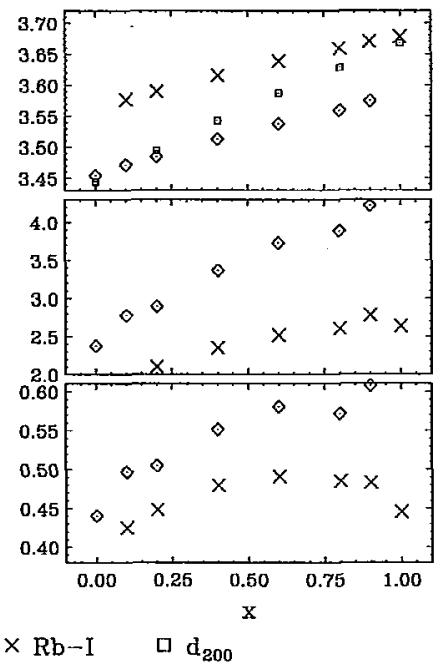

Fig. 2. Comparison of EXAFS-extracted (left) and MD-extracted (right) structural parameters in $\operatorname{RbBr}_{1-\mathrm{x}} \mathbf{I}_{\mathbf{x}}$ alloys. In subsequent rows the $x$-dependences of $R, \sigma^{2}$ and $\beta$ values are compared

As one can see, the simulated average lattice constants $\left(\mathrm{d}_{200}\right)$ are in fairly good quantitative agreement with the experimental data (difference not larger than 0.3\%). As expected, it depends linearly on the stoichiometry (Vegard's law). The lattice constants were reproduced with less accuracy only for pure RbI and the alloys with stoichiometry close to this salt $(0.7 \%)$. Too small values of this parameter result in densities of MD-simulated samples somewhat larger than the experimentally measured ones.

The simulated cation-anion distances change and depart from the average distance between the layers as the content of the second anion increases less rapidly than it has been observed experimentally. The slope of the $\mathrm{Rb}-\mathrm{X}(\mathrm{X}=\mathrm{Br}, \mathrm{I})$ bond length versus $x$ plot seems to be constant and is greater than the one derived from the experimental data. Consequently, in the simulated compounds, the difference between the $\mathrm{Rb}-\mathrm{Br}$ and $\mathrm{Rb}-\mathrm{I}$ distances is smaller than in the real materials (less than 2\%). 
The width of RDF peaks for each sample increases with the increase of iodine content. In the whole range of $x$ the width, of the first $\mathrm{Rb}-\mathrm{Br}$ peak is grater than that of the $\mathrm{Rb}$-I peak. It is in qualitative agreement with the experimental data. Values for the Rb-I pair have been reproduced with worse quantitative agreement.

It is difficult to specify any tendency in dependence of the asymmetry of the peaks on the stoichiometry. The values obtained for the Rb-I pair vary in the same range; in the simulation the asymmetry increases as the compound stoichiometry departes from pure $\mathrm{RbBr}$ or $\mathrm{RbI}$ systems. In the case of $\mathrm{Rb}-\mathrm{Br}$ peaks the asymmetry observed in the simulations is greater than that of the $\mathrm{Rb}$-I correlation, and it increases with iodine concentration. At this stage of analysis of the EXAFS data, the values of $\beta$ are considered to be constant, i.e. stoichiometry-independent. The difference between the values obtained in the MD simulations and the experimental measurements is not greater than $5 \%$.

\section{CONCLUSIONS}

In the present contribution, the potential parameterisations proposed by Dixon and Sangster [10] for ionic $\mathrm{RbBr}$ and $\mathrm{RbI}$ salts have been applied to simulate the structure of ternary $\operatorname{RbBr}_{1-x} \mathrm{I}_{x}$ alloys. From a comparison with the experimental data extracted from the X-ray diffraction and the EXAFS analysis, one can see that the structural parameters, as well as their variations in the function of the system stoichiometry, are in qualitative agreement. For most samples, the lattice constants are reproduced with very good quantitative agreement and obey the Vegard's law. The cation-anion distances, as expected, depart from the average lattice constant. However, these changes are somewhat smaller than the ones observed in the EXAFS experiment. Due to the difference of RbI's density in comparison with the experimental one (simulations were performed in the $\mathrm{NpT}$ ensamble), the $\mathrm{Rb}-\mathrm{I}$ bond lengths obtained for all compounds have been shorter than the ones extracted from the analysis of the EXAFS signal. It should be mentioned, that similar MD simulations performed by us using the interactions proposed in [12] have yielded results in much worse agreement with the presented experimental data.

Classical molecular dynamics simulations of ionic alloys are a powerfull tool to study their structural properties. Although the potential parameterisation of Dixon and Sangster has been quite successfully applied to the $\operatorname{RbBr}_{1-x} I_{x}$ alloys, further work on interatomic potentials should be undertaken. The new interaction model should better reproduce the material densities, and lead to greater bond-length mismatch. On the other hand, the EXAFS-extracted data also require further refinement.

\section{Acknowledgement}

The simulations have been performed at the TASK Computer Centre in Gdańsk (Poland). 


\section{References}

[1] A. Di Cicco, E. Principi, A. Filipponi, Phys. Rev., B65, 212106 (2002).

[2] M. F. Thorpe, E. J. Garboczi, Phys. Rev., B42, 8405 (1990).

[3] Y. Cai, M. F. Thorpe, Phys. Rev., B46, 15872 (1992).

[4] Y. Cai, M. F. Thorpe, Phys. Rev., B46,15879 (1992).

[5] J. C. Mikkelsen, J. B. Boyce, Phys. Rev., B28, 7130 (1983).

[6] J. B. Boyce, J. C. Mikkelsen, Phys. Rev., B31, 6903 (1985).

[7] A. Filipponi, A. Di Cicco, C. R. Natoli, Phys. Rev., B52, 15135 (1995).

[8] A. Filipponi, A. Di Cicco, Phys. Rev., B52,15122 (1995).

[9] A. Di Cicco, Phys. Rev., B53, 6174 (1996).

[10] M. Dixon, M. J. L. Sangster, J. Phys. C: Solid State Phys., 9, 3381 (1976).

[11] http://www.dl.ac.uk/TCSC/Software/DL_POLY/main.html

[12] A. Baranyai, I. Ruff, R. L. McGreevy, J. Phys. C: Solid State Phys., 19(4), 453 (1986). 